\title{
When two kinases are better than one
}

$\mathrm{V}$

idal-Eychenié et al. describe how two protein kinases team up to respond to DNA damage at stalled replication forks.

DNA-dependent protein kinase catalytic subunit (DNA-PKcs) promotes the repair of DNA double-strand breaks (DSBs) by nonhomologous end joining (NHEJ). The ATR kinase, on the other hand, initiates cell cycle arrest and DNA repair after recognizing the long stretches of single-stranded DNA (ssDNA) that accumulate when replication forks are delayed. In cancer cells, which are under increased replicative stress, stalled replication forks collapse to generate DSBs as well as ssDNA, but whether DNA-PKcs and ATR cooperate to initiate a DNA damage response at these lesions is unknown.

Vidal-Eychenié et al. emulated the damage at collapsed replication forks by designing DNA fragments containing a short stretch of ssDNA and double-stranded ends that mimic DSBs. These

\section{Lis 1 puts mRNAs in their place}

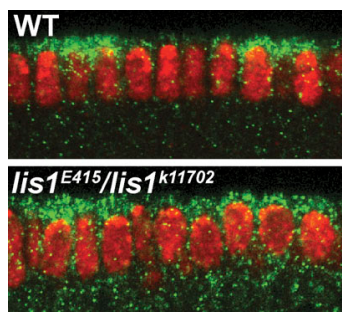

The apical accumulation of hairy RNA (green) is reduced in an embryo lacking Lis 1 (bottom) compared with a control embryo (top). Nuclei are labeled red.

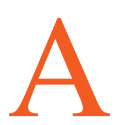

protein associated with the neurodevelopmental disorder lissencephaly promotes the assembly of dynein-dynactin motor complexes on mRNA cargoes, Dix et al. reveal.

Many mRNAs are transported to specific locations within the cell so that the proteins they encode are synthesized in the right place. In early Drosophila embryos, for example, certain mRNAs are transported to the apical cytoplasm by the minus end-directed microtubule-based motor dynein and its accessory complex dynactin. Dynein and dynactin are recruited to their cargo by adaptor proteins that bind to a "localization signal" in the mRNA. How the assembly and function of the motor complex are regulated is unclear, however.

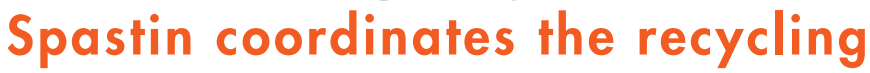

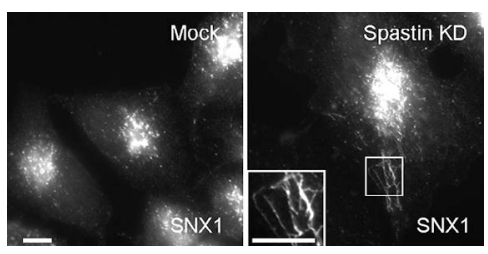

The endosomes (white) of a cell lacking spastin (right) are highly tubulated compared with those in control cells (left) because tubule fission is impaired.

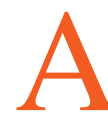

llison et al. reveal how a protein linked to hereditary spastic paraplegia (HSP) promotes the traffic of endosomal tubules that recycle proteins back to the cell surface.

Mutations in the gene encoding the microtubule-severing protein spastin cause a dominant form of HSP. Spastin helps remodel membranes at the ER, where it interacts with several proteins that shape membrane tubules, and at the midbody, where it promotes cytokinesis. Spastin also localizes to endosomes, but its function at this organelle is unknown. In wild-type cells, plasma membrane proteins are recycled to the cell surface in membrane tubules that pinch off from sorting endosomes, leaving behind proteins that, after being internalized fragments induced a robust DNA damage response when incubated in cell-free extracts, but this response was blocked by inhibitors of both DNA-PKcs and ATR. DNA-PKcs enhanced ATR's activity by phosphorylating two proteins-RPA32 and TopBP1 - that aid the kinase's recruitment and activation on short stretches of ssDNA. This might help ATR respond immediately to replication catastrophes.

ATR, in turn, promoted the phosphorylation of DNA-PKcs at a site that might cause the kinase to favor the repair of DSBs by homologous recombination instead of NHEJ, thereby reducing genome instability. DNA-PKcs and ATR therefore act synergistically to respond to replication-associated damage in cancer cells. Senior author Angelos Constantinou says that inhibitors of this pathway could be used to selectively target tumor cells in vivo.

Vidal-Eychenié, S., et al. 2013. J. Cell Biol. http://dx.doi.org/10.1083/ jcb.201304139.

Dix et al. found that the dynein-associated protein Lis1 was recruited to the localization signals of several Drosophila mRNAs. Fly embryos with reduced levels of Lis1 were defective in transporting these mRNAs to the apical cytoplasm, because their movement toward the minus end of microtubules was impaired. Defects in RNA transport could contribute to the developmental problems associated with lissencephaly, in which the gene encoding Lis1 is mutated.

Dynein transports numerous cellular cargoes, and Lis1 promotes the movement of many, if not most, of them by regulating dynein's motor activity. This may also be true for the apical transport of mRNAs, but Dix et al. found that Lis1 also promoted dynein's association with the dynactin complex and the recruitment of these proteins to RNA localization signals. Senior author Simon Bullock now wants to investigate how Lis1 promotes the assembly of the dynein-dynactin complex and to determine whether it remains associated with mRNAs once they start to move.

Dix, C.I., et al. 2013. J. Cell Biol. http://dx.doi.org/10.1083/jcb.201211052.

\section{program}

into vesicles inside the endosome lumen, are transported to lysosomes to be degraded.

Allison et al. found that, in the absence of spastin, recycling tubules failed to separate from sorting endosomes, prompting cargo such as the transferrin receptor to be degraded instead of returned to the plasma membrane. Tubule fission wasn't restored by spastin mutants unable to sever microtubules or by versions of the protein unable to bind IST1, a component of the ESCRT-III complex that sorts endosomal cargo into intralumenal vesicles. Cells lacking IST1 showed similar defects in tubule fission and transferrin receptor trafficking, suggesting that IST1 recruits spastin to coordinate the recycling and degradation of endosomal proteins.

Defects in this process may give rise to HSP. Knocking down spastin or IST1 inhibited the growth of zebrafish motor axons. The endosomes in these neurons were abnormally tubulated, which could, says senior author Evan Reid, affect the trafficking of signaling proteins like the BMP receptor that are crucial for axon development. Allison, R., et al. 2013. J. Cell Biol. http://dx.doi.org/10.1083/jcb.201211045. 